\title{
Impactos del turismo sobre el crecimiento económico y el desarrollo. El caso de los principales destinos turísticos de Colombia*
}

\author{
Juan Gabriel Brida ${ }^{i}$ \\ University of Bolzano (Italia) \\ Pablo Daniel Monterubbianesii ${ }^{i i}$ \\ Universidad Nacional del Sur (Argentina) \\ Sandra Zapata-Aguirre ${ }^{\mathrm{iii}}$ \\ Institución Universitaria Colegio Mayor de Antioquia (Colombia)
}

\begin{abstract}
Resumen: Este trabajo analiza el rol del turismo y sus efectos sobre el crecimiento económico y el desarrollo, centrándose en cuatro importantes regiones turísticas de Colombia. Para el análisis se estudian las relaciones entre turismo y crecimiento y desarrollo económico desde dos ópticas distintas y complementarias. En primer lugar se cuantifica la contribución del sector turismo al crecimiento de la economía desde el año 1990 hasta el 2006. En segundo lugar, se estudia la importancia que tiene el turismo en el crecimiento y desarrollo económico de largo plazo. Los análisis realizados muestran una participación creciente del turismo en el crecimiento económico de cada región durante los periodos 1990-1999 y 2003-2006 y decreciente en el periodo intermedio. Se muestra una clara causalidad positiva y unidireccional desde el gasto en turismo hacia el producto per cápita en cada región, pero también diferencias significativas en los valores de las elasticidades entre las variables consideradas. Esto implica que un incremento del gasto turístico va a provocar efectos diferentes en el crecimiento económico de cada destino.
\end{abstract}

Palabras clave: Gasto en turismo, Crecimiento Económico, Desarrollo, Cointegración de Johansen, Elasticidad, Causalidad de Granger, Colombia.

Title: The impacts of tourism on economic growth and development: the case of the main Colombian destinations

Abstract: This paper analyses the effects of tourism on the economic growth and development, focusing on the main tourism regions of Colombia: Antioquia, Bolivar, Bogotá, Magdalena and San Andres-Providencia of Colombia. For the analysis, the study uses annual data from 1990 to 2006. Firstly, the work presents a quantitative exercise describing the evolution of the participation of the tourism sector in the different regions and its contribution to economic growth. The analysis shows an increasing participation of tourism in the economic growth of each region during the periods 19901999 and 2003-2006 and a decresing in the intermediate period. Secondly, the study uses cointegration analysis to evaluate the existence of VEC among real GDP per capita, tourism expenditures and real exchange rates. We show that the causality relationship is positive and unidirectional for all the regions but the values of elasticities are considerable different. This implies that a change in tourism expenditure will produce different effects on the economic growth of the regions.

Keywords: Economic growth; Tourism earnings; Johansen cointegration test; Vector Error Correction; Colombia.

i Competence Centre in Tourism Management and Tourism Economics (TOMTE). School of Economics and Management-Free University of Bolzano, Italia. E-mail: JuanGabriel.Brida@unibz.it

ii Departamento de Economía, Universidad Nacional del Sur. Argentina. E-mail: pmonteru@uns.edu.ar.

iii Grupo de Investigación Empresarial y de Turismo (GIET). Institución Universitaria Colegio Mayor de Antioquia. Colombia. E-mail: sandra.zapata@colmayor.edu.co 


\section{Introducción}

Reconocido como un sector industrial que tiene un impacto positivo sobre el crecimiento y el desarrollo económico (Kim et al., 2006; Balaguer y Cantavella, 2002; Capó et al, 2007A y 2007B; Noriko and Mototsugu, 2007; Dritsakis, 2004; Durbarry, 2004; Meyer, 2004), El turismo es un rama de la actividad económica que representa aproximadamente el 10\% del PIB mundial y esta participación viene creciendo en los últimos decenios. Las cifras que anualmente registra el ingreso mundial por turismo internacional refleja la importante contribución a la dinámica económica que esta actividad representa para muchas regiones en el mundo. Entre sus principales impactos positivos encontramos los relacionados con el ingreso por divisas, la contribución al ingreso privado y público, la generación de empleo, el incentivo a la creación de tecnologías y a la formación de capital humano y las oportunidades de negocio que puede generar en una economía. Estos beneficios han llevado a este sector económico a ganarse un puesto protagónico en el ámbito mundial y desplazar a sectores tradicionales en diversos destinos. En particular, en Colombia el turismo ha venido registrando un destacado ascenso que no experimentaba desde hace décadas. La evolución de la participación del turismo en el PIB nacional ha mostrado una tendencia a la recuperación, pasando de un $2,21 \%$ en 2000 a 2,32\% en 2005 . La visión para 2011 está encaminada a incrementar en un 100\% el nivel actual de ingresos por turismo. Las cifras de empleo generado por el sector correspondiente a hoteles, restaurantes y agencias de viajes registraron un crecimiento del orden de $31,64 \%$ en el periodo $2001-2005$ pasando de 50.154 puestos de trabajo a $66.022^{1}$. De todos modos, es de destacar que las derramas económicas, laborales y sociales que el turismo genera, se vuelcan mayoritariamente a las regiones que tienen atractivos turísticos. Es por esa razón que en este trabajo hemos elegido como estudio de caso los departamentos de Colombia más importantes desde el punto de vista del turismo.

El turismo puede transformarse en un importante y dinámico promotor del desarrollo, pero al mismo tiempo representa el desafío para todos los actores implicados de buscar soluciones a los problemas que se pueden generar y para encauzar y orientar el desarrollo del turismo. Las Administraciones Públicas desempeñan un papel central en el logro de una mayor competitividad del sector, favoreciendo la implantación de un marco adecuado para que la actividad turística sea competitiva, y busque el equilibrio necesario entre intereses, garantizando que los impactos negativos que provoca el turismo sean mínimos. Esto exige introducir una visión de largo plazo en el desarrollo de la actividad turística que se vea reflejada en una adecuada política turística. El turismo impacta en el desarrollo regional en la medida que (1) genera cambio económico a través del crecimiento del empleo, del crecimiento de la producción directa, indirecta e inducida, de la transformación de los rubros económicos tradicionales, de la mejora de los recursos públicos y de la distribución de la renta, entre otros factores; (2) genera efectos sociales y ambientales a través de la diversificación del empleo, del mejoramiento de infraestructuras y de los desajustes socioculturales, brindando posibilidades de movilidad social, etc. (Figuerola, 2005). Las políticas turísticas del desarrollo regional deben contemplar en términos generales, la subvención al desarrollo, la accesibilidad crediticia, el mejoramiento del sistema de transporte, el desarrollo de tecnologías, el desarrollo estadístico, el ordenamiento territorial y el mejoramiento de la seguridad. Desde la especificidad del turismo, la promoción y el marketing, la investigación, la financiación de programas, la diversificación de la oferta, el desarrollo de actividades complementarias, la formación y mentalización social, la conservación y recuperación del patrimonio y la puesta en valor de recursos.

A nivel regional, se reconoce la importancia del turismo como un elemento dinamizador de las economías locales. Dicho reconocimiento se manifiesta como una consideración importante en los planes de desarrollo tanto del orden nacional como regional. Prevalece en éstos, los esfuerzos por invertir en promoción y diseño de nuevos productos turísticos, investigación de mercados, infraestructura y estímulos a la creación de nuevas prestadores de servicios turísticos. Esfuerzos que, si bien valiosos para un importante grupo en el turismo, no resultan útiles al momento de evaluar la forma en que el turismo contribuye a la economía en su conjunto. Dada la importancia que tiene esta actividad en muchas regiones, es necesario determinar la real dimensión que ese impacto económico genera en ellas, asimismo, evaluar cómo influye el turismo en el crecimiento de largo periodo. Se requiere entonces un mayor interés por parte de los órganos decisores (no sólo del orden gubernamental sino también privado) en el análisis de los diferentes costos e impactos de esta actividad. Estos impactos económicos se clasifican en tres tipos de impactos: directos, indirectos e inducidos. La principal dificultad al medir el impacto económico del turismo se debe al carácter multisectorial de la actividad turística que conlleva beneficios que resultan difíciles de cuantificar y que de alguna manera disminuyen la verdadera contribución del turismo al desarrollo económico (Meyer, 2004). Por otro lado, debido a la inexistencia de un instrumento estadístico 
nacional que proporcione dichos datos y además, que gocen de credibilidad. A nivel nacional, en Colombia aún no existe una cuenta satélite y a nivel regional menos cierto. Estos mecanismos le proporcionarían a los órganos decisores que participan en el turismo un mejor conocimiento en relación con el papel que desempeña el turismo en sus regiones y más específicamente a la contribución de éste en el crecimiento económico. Entendimiento que se pretende sea útil en la elaboración de políticas económicas coherentes con los niveles de desarrollo que pueda generar la actividad en sus regiones. La OMT se ha comprometido a ofrecer a sus Miembros la asistencia técnica necesaria para que puedan cuantificar el número de empleos que genera el turismo en sus respectivas economías, la contribución del sector al PIB, y la posición de la industria turística con respecto a otros sectores de la economía (OMT, 2009).

En este artículo estudiamos las relaciones entre turismo y crecimiento y desarrollo económico desde dos ópticas distintas y complementarias. En primer lugar se cuantifica la contribución del sector turismo al crecimiento de la economía desde principio de la década del 90 hasta el año 2006. Se trata de un análisis descriptivo que mide lo que efectivamente sucedió en esos años. En segundo lugar, se estudia la importancia que tiene el sector turismo en el crecimiento y desarrollo económico de largo plazo. Esto es, se trata de una metodología que pretende hacer una previsión del impacto del sector turístico en la economía de cada una de las regiones consideradas. Con dicho objetivo, se recurre a las técnicas de cointegración desarrolladas por (Johansen, 1988), y a la estimación de modelos con mecanismos de corrección del error (Vectores Autorregresivos con Mecanismo de Corrección del Error). Estas técnicas permiten determinar la existencia de una relación de equilibrio en el largo plazo entre las variables que son objeto de estudio, y modelar concomitantemente la dinámica de largo y corto plazo que las vincula. A su vez, se analiza la dirección de la causalidad (en el sentido de Granger) entre el gasto en turismo y el crecimiento económico de largo plazo.

Con respecto al primer enfoque, en un artículo reciente Ivanov y Webster (2007) presentan una metodología para medir la contribución del turismo al crecimiento económico. El método utiliza el crecimiento real del PIB per cápita como una medida de crecimiento económico el cual se desglosa en un crecimiento económico generado por el turismo y el crecimiento económico generado por otras industrias. La metodología tiene la particularidad de dar una estimación a posteriori de la contribución del turismo sobre el PBI, siendo un instrumento que complementa análisis más sofisticados. Esta metodología ha sido aplicada a los datos de distintos países y regiones pero no existen estimaciones para el caso de las regiones bajo estudio en este trabajo.

La segunda metodología que se introduce en este trabajo permite investigar relaciones causales entre el PIB per cápita, el gasto en turismo y la tasa de cambio real. Esto nos permite concluir si alguna de las variables puede causar las otras permitiendo extraer conclusiones acerca del potencial de desarrollo económico en las regiones de Colombia bajo estudio, especialmente en lo que tiene que ver con el turismo como sector económico y su contribución al crecimiento. Estas hipótesis son testeadas mediante el test de cointegración de Johansen y el test de causalidad de Granger. Estas técnicas se basan en los trabajos Engle y Granger (1987), Granger (1987), Johansen (1988 y 1995) y Johansen y Juselius (1990).

El resto del artículo se organiza de la siguiente forma. En la siguiente sección presentamos algunos antecedentes sobre la evolución económica del sector turismo, progresos y principales cambios de política económica del turismo en las regiones bajo estudio. En la sección tres se discuten la metodología y la evidencia empírica. Finalmente, en la última sección se presentan las conclusiones y futuras líneas de investigación.

\section{Características del sector turismo en las princi- pales regiones colombianas}

En este trabajo hemos seleccionado los departamentos de Colombia en los cuales el turismo tiene mayor presencia, en comparación con los demás, Es así que siguiendo los datos presentados por el Departamento Administrativo Nacional de Estadística (DANE), fueron elegidos los siguientes destinos: Antioquia, Bogotá (Distrito Capital), Bolívar, Magdalena y San Andrés-Providencia. A continuación presentamos las principales características del turismo en cada una de estas regiones.

\section{Departamento de Antioquia}

Localizado en la zona noroccidental del país, Antioquia es uno de los departamentos colombianos cuyo impulso económico está repartido principalmente en las siguientes ramas de la actividad económica: industria; servicios sociales, comunales y personales; comercio, hoteles y restaurantes; construcción y transporte; agropecuario, silvicultura y pesca. El departamento de Antioquia concentró $14,3 \%$ del total de las exportaciones nacionales en 2005. A éste le siguieron el departamento de Cundinamarca con un $8,5 \%$, Bogotá D.C con un $8,2 \%$ y Valle del Cauca con un $7,7 \%^{2}$. El departamento, al igual que las otras regiones en estudio, cuenta con 
importantes recursos de toda índole, susceptibles de ser aprovechados turísticamente pero que carece de una sólida voluntad pública y privada para maximizar dichos potenciales. Es un departamento con un desarrollo turístico incipiente, básicamente a causa de un desarrollo institucional y organizacional débil desde lo público, acompañado de un sector privado con bajos resultados en productividad y competitividad (Gobernación de Antioquia). El departamento tiene como meta para 2011 lograr una participación porcentual del sector turístico en el PIB del Departamento del orden del 2,04\%. En su capital, la ciudad de Medellín, está concentrada la mayor actividad turística de la región y es, entre las principales ciudades del país, la que ofrece una mejor plataforma para desarrollar negocios de acuerdo con un informe contratado por las principales cámaras de comercio nacionales ${ }^{3}$. En esta ciudad se desarrolla básicamente el producto de turismo de negocios.

\section{Bogotá Distrito Capital}

Bogotá es la ciudad capital de Colombia y del departamento de Cundinamarca. Está ubicada en el centro del país sobre una meseta de la Cordillera Oriental de los Andes a $2.630 \mathrm{msnm}$. Bogotá es una ciudad que ha experimentado una positiva transformación durante los últimos 10 años. La ciudad es hoy reconocida a nivel internacional por múltiples iniciativas que han dado como resultado el mejoramiento del equipamiento urbano, el aumento en la seguridad ciudadana y en general, en una mayor calidad de vida de los habitantes. Entre esos reconocimientos se pueden mencionar: (1) Líder en prestación de servicios médicos especializados, según la Organización Mundial de la Salud. (2) Capital Iberoamericana de la Cultura 2007: Esta distinción concedida por la UCCI (Unión de Ciudades Capitales Iberoamericanas) reconoce la transformación de la ciudad en los últimos años y su liderazgo en cultura ciudadana, cumplimiento individual de la ley, autorregulación y convivencia, entre otros ${ }^{4}$. Bogotá es el principal centro económico e industrial de Colombia. Así mismo, es uno de los polos financiero e industrial importantes en América Latina. El distrito capital tiene en los servicios financieros, los servicios sociales, la industria y el comercio, su base económica. El PIB comercial de Bogotá representó, durante el periodo 2003-2006, el 25,3\% del PIB comercial nacional (Secretaría distrital de Planeación). Si bien es cierto que Bogotá es una urbe de negocios y de política, también es importante resaltar su gestión en el desarrollo cultural del país y turístico del país. Según cifras del Ministerio de Comercio, Industria y Turismo ${ }^{5}$, la ciudad ocupa el primer lugar en recepción de visitantes extranjeros con una participación de
38,3\% del total del turismo internacional que llega vía aérea al país.

\section{Departamento de Bolívar}

Está ubicado al norte del país y forma parte de la Región Caribe. La base económica de este departamento está concentrada en su capital, la ciudad de Cartagena de Indias donde se desarrollan las actividades de tipo industrial, turística y portuaria. El resto de los municipios que conforman el departamento, basan su economía esencialmente en actividades agropecuarias, mineras y artesanales (Pérez, 2005). Es así que, al hablar de turismo, se hace referencia a los datos estadísticos sobre la participación de hoteles y restaurantes fundamentalmente de dicha capital. No obstante, el resto del departamento cuenta con numerosos recursos que le permitirían ampliar su oferta de productos turísticos. La promoción turística internacional de esta región está basada en el producto historia y cultura, representado en el centro histórico de su capital, Cartagena de Indias, cuyo valor la hace parte del conjunto de ciudades patrimonio de la humanidad. A nivel nacional, esta ciudad es líder con el producto de sol y playa entre las ciudades del Caribe continental nacional y recibe actualmente el $14 \%$ del turismo internacional. Cartagena de Indias, según diferentes indicadores de competitividad aplicados en el trabajo (Quintero et al, 2005) es uno de los destinos con mayor competitividad turística en el mercado nacional. Bolívar, después de San Andrés-Providencia y Magdalena, es el departamento en el que el turismo tiene mayor participación sobre su PIB.

\section{Departamento de Magdalena}

Ubicado en la Región Caribe, al norte del país. Su actividad económica se sustenta en las actividades agropecuarias, ganaderas, turísticas y portuarias. El turismo se concentra en Santa Marta y el Parque Tayrona, mientras que el comercio y los servicios están solo en la capital. El aporte de los hoteles y restaurantes al PIB en el departamento del Magdalena es de 6,3\% (DANE); indudablemente, su capital, Santa Marta, contribuye con la mayor parte. Siendo éste el tercer destino de preferencia de los colombianos $(11,5 \%)$, y con mayor promedio de pernoctación 11.1 noches, frente a un promedio nacional de (9) nueve (DANE). De acuerdo con la gobernación departamental, Magdalena y su Capital Santa Marta, son un compendio de atractivos de todo tipo: de clima seco, de sol y playa, de montañas, de nevados, de ríos cristalinos, de aguas termales, de lugares arqueológicos, actividades eco-turísticas, etc., pero que debido a su débil infraestructura básica para su acceso le hacen perder competitividad frente a otros destinos naciona- 
les que reciben turismo internacional. En el documento conceptual para la elaboración de su próximo Plan Sectorial de Turismo, los actores involucrados refieren que el departamento carece de acciones que le ayuden a mejorar sus servicios turísticos. Que la región requiere mayores inversiones públicas y privadas que se desarrollen como resultado de las políticas nacionales y distritales, que orienten la competitividad y sostenibilidad al sector. A través de dicho plan se pretende consolidar el turismo como el sector de mayor importancia en el desarrollo económico y social de la ciudad, dada su contribución en el crecimiento de la producción, el ingreso local y la capacidad de generar empleos de calidad que aportan a la calidad de vida de las comunidades ${ }^{6}$.

\section{Departamento de San Andrés-Providencia}

El Archipiélago es el Departamento más grande del país, ubicado al Noroeste de Colombia localizado al occidente de la llamada "Región del Gran Caribe" sobre el mar de las Antillas ${ }^{7}$. La capital departamental es San Andrés, pero el Archipiélago tiene un sólo municipio, Providencia. El departamento limita con cinco países del área de Centroamérica y del Caribe. En el año 2000 fue declarada Reserva de la Biosfera Seaflower por la UNESCO. La economía del Departamento de San Andrés y Providencia está basada principalmente en el turismo y el comercio, que siguen generando la gran mayoría de las nuevas empresas. Otras actividades que complementan la base económica son la agricultura y la pesca (Banco de la República, 2006). Con la declaración del puerto libre, y las migraciones posteriores tanto de la población del interior (Bolivar, Atlántico, Antioquia) como extranjeros (Medio Oriente) se impulsó la actividad turística y comercial, incentivadas por los bajos costos que tenían las mercancías. Con ello, aumentó el transporte aéreo y marítimo a la isla, que durante las temporadas altas es visitada por una gran cantidad de turistas.

\section{Metodología}

El objetivo de esta sección es describir las metodologías a aplicar. En primer lugar, se expone el método utilizado para estimar la contribución del sector turismo en el crecimiento económico. En segundo lugar, se presenta la metodología que se empleará para el estudio de la importancia que tiene el sector turismo en el crecimiento de largo plazo de la economía colombiana.

\section{Contribución del turismo al crecimiento económico.}

Tradicionalmente, una gran proporción del gasto turístico tiene como destino no sólo a los sectores ca- racterísticos del turismo como el transporte, los hoteles y la recreación, sino también a otros sectores que no están normalmente asociados con el turismo. Teniendo en cuenta que la contribución económica del turismo está repartida entre una serie de distintos sectores es, por consiguiente, difícil cuantificar la contribución del turismo al crecimiento económico de una economía. Esta es la primera limitación de nuestro ejercicio ya que, al utilizar los datos de los sistemas de cuentas nacionales, vamos a considerar como "turismo" sólo a los sectores relacionados directamente con él (específicamente, hoteles y restaurantes). Una medida muy usada para estimar la magnitud del turismo como sector económico es la de porcentaje de participación del sector en el PIB, es decir, qué porcentaje del PIB corresponde al turismo. Nótese que esta medida nada dice sobre cuánto contribuye el sector turismo al crecimiento económico sino que sólo indica para cada nivel de PIB cuánto corresponde al turismo. Como en la contribución de Ivanov y Webster (2007), en este trabajo se utilizará la tasa de crecimiento del PBI real per cápita ( ), como medida del crecimiento económico:

$$
g_{r}=\left(\frac{\frac{\sum_{t} Y_{r\left(p_{0}\right)}^{t}}{N_{r}}-\frac{\sum_{t} Y_{r-1\left(p_{0}\right)}^{t}}{N_{r-1}}}{\frac{Y_{r-1\left(p_{0}\right)}}{N_{r-1}}}\right)
$$

donde $\quad \sum_{t} Y_{r\left(p_{0}\right)}^{t}$ es el PIB total de la economía en el periodo $r$ a los precios $\mathrm{p}_{0}$ (precios constantes) y $N$ es la población en el periodo $r$. Entonces, desglosando el PIB del turismo del resto de la economía, obtenemos:

$$
g_{r}=\left(\frac{\frac{Y_{r\left(p_{0}\right)}^{T}}{N_{r}}-\frac{Y_{r-1\left(p_{0}\right)}^{T}}{N_{r-1}}}{\frac{Y_{r-1\left(p_{0}\right)}}{N_{r-1}}}+\frac{\frac{\sum_{t \neq T} Y_{r\left(p_{0}\right)}^{t}}{N_{r}}-\frac{\sum_{t \neq T} Y_{r-1\left(p_{0}\right)}^{t}}{N_{r-1}}}{\frac{Y_{r-1\left(p_{0}\right)}}{N_{r-1}}}\right)
$$

donde el primer componente en esta expresión:

$$
g_{r}^{T}=\left(\frac{\frac{Y_{r\left(p_{0}\right)}^{T}}{N_{r}}-\frac{Y_{r-1\left(p_{0}\right)}^{T}}{N_{r-1}}}{\frac{Y_{r-1\left(p_{0}\right)}^{T}}{N_{r-1}}}\right)
$$

representa la contribución directa de la industria del turismo en el crecimiento económico en el periodo r. Nótese que $g_{r}^{T}$ mide la variación del PIB producida por el sector turístico. 


\section{La hipótesis del turismo como generador de desamo- llo y crecimiento de largo periodo}

Posteriormente, analizamos la relación de largo plazo entre el gasto en turismo y el producto real per cápita. Para ello emplearemos las siguientes variables:

1. Gasto en turismo (TE): gasto en el sector turismo (hoteles y restaurantes) en millones de pesos colombianos a precios de 1994 .

2. PBI/L: producto per cápita neto de impuestos en pesos colombianos a precios de 1994.

3. TCR: tipo de cambio real, estimado por el Banco Central de Colombia (Banco de la República) en comparación con 21 países que interactúan con este país.

En economía (y en particular en los análisis econométricos) es muy frecuente encontrarse con el concepto de regresión espuria, el cual se da cuando tenemos una relación entre dos variables, con una alta significatividad y un elevado $R^{2}$ pero que en realidad no es tal, sino que el valor de estos indicadores se debe a que las series bajo consideración son no estacionarias, de forma tal que los mencionados test pierden validez. Sin embargo, cuando tenemos series no estacionarias que son integradas del mismo orden, podemos tener una combinación lineal de ellas que sea estacionaria, de forma tal que los test retoman su validez. En este caso, se dice que estas variables se encuentran cointegradas. De esta forma, para comenzar el análisis, debemos analizar la estacionariedad de las series bajo consideración y el grado de integración de las mismas. Para poder analizar la estacionariedad de las series emplearemos el test de DickeyFuller Aumentado (ADF), el cual plantea como hipótesis nula la existencia de una raíz unitaria. El test aplicado se basa en la técnica propuesta por Dolado et al. (1990), la cual nos permite emplear el test ADF y al mismo tiempo evitar el problema de potencia que el mismo presenta.

En caso de existir una relación de cointegración entre las variables, podremos sostener que existe una relación de largo plazo entre las mismas, la cual se puede modelar mediante un modelo de corrección de errores (VEC). Banerjee et. al. (1993) señalan la importante conexión que existe entre una relación de cointegración y la correspondiente relación de equilibrio de largo plazo. Buscar una relación de cointegración es buscar un equilibrio estadístico entre variables que tienden a crecer en el tiempo. Todo lo que se aparta del equilibrio puede ser modelado por un Vector de Corrección de Errores (VCE) que muestra como después de un shock las variables regresan al equilibrio. Para testear la existencia de relacio- nes de cointegración, emplearemos la técnica planteada por Johansen (1988) y Johansen y Juselius (1990), que evalúa simultáneamente todas las relaciones de cointegración posibles. Al construir el modelo de corrección de errores, podremos también estimar la elasticidad del ingreso respecto al gasto en turismo, información que nos permitirá evaluar el efecto de cambios en el gasto turístico sobre el crecimiento económico.

\section{Evidencia empírica}

En esta sección, se presentan los resultados de las estimaciones que surgen de aplicar las metodologías propuestas al conjunto de datos mencionados. En cuanto a los datos cabe aclarar que las series temporales cuatrimestrales del Producto Bruto Interno (PBI) real, las series anuales para la población, el gasto anual real en hoteles y restaurantes y los datos anuales del consumo final se obtuvieron del Departamento Administrativo Nacional de Estadística (DANE). Finalmente, la serie de tipo de cambio real entre el peso colombiano y las monedas de 18 países que es utilizada en el artículo, proviene del Fondo Monetario Internacional.

\section{Resultados del análisis ex-post}

Los resultados de la aplicación de la metodología se presentan en los siguientes cuadros (Fuente: Elaboración propia con base en datos del DANE), en los que se indica para cada año, la variación real del PBI per cápita, variación real del PBI per cápita del sector turístico (medido a través del sector de hoteles y restaurantes) y

\begin{tabular}{|l|r|r|r|r|r|r|r|r|}
\hline & 1990 & 1991 & 1992 & 1993 & 1994 & 1995 & 1996 & 1997 \\
\hline Colombia & 2,76 & 2,78 & 2,80 & 2,72 & 2,65 & 2,68 & 2,48 & 2,26 \\
\hline Antioquía & 2,15 & 2,04 & 2,13 & 2,08 & 2,07 & 1,98 & 1,79 & 1,69 \\
\hline Bogotá & 1,80 & 1,86 & 1,96 & 1,95 & 1,88 & 2,12 & 2,01 & 1,81 \\
\hline Bolívar & 6,17 & 6,68 & 6,44 & 6,01 & 5,80 & 6,00 & 5,73 & 3,68 \\
\hline Magdalena & 6,09 & 6,39 & 6,29 & 6,01 & 6,12 & 6,08 & 5,92 & 5,63 \\
\hline San Andrés & 19,46 & 17,12 & 16,78 & 20,80 & 21,22 & 21,72 & 23,21 & 23,77 \\
\hline & & & & & & & & \\
\hline & 1998 & 1999 & 2000 & 2001 & 2002 & 2003 & 2004 & 2005 \\
\hline Colombia & 2,27 & 2,22 & 2,21 & 2,22 & 2,21 & 2,30 & 2,32 & 2,32 \\
\hline Antioquía & 1,74 & 1,59 & 1,59 & 1,69 & 1,62 & 1,64 & 1,69 & 1,71 \\
\hline Bogotá & 1,96 & 1,91 & 1,99 & 1,97 & 1,99 & 2,28 & 2,26 & 2,28 \\
\hline Bolívar & 3,35 & 3,77 & 4,18 & 4,34 & 4,08 & 4,34 & 4,31 & 4,52 \\
\hline Magdalena & 5,62 & 5,79 & 6,04 & 6,33 & 6,50 & 6,46 & 6,53 & 6,57 \\
\hline San Andrés & 22,08 & 26,61 & 24,41 & 31,45 & 29,73 & 26,50 & 26,92 & 26,88 \\
\hline
\end{tabular}

Cuadro 1: Porcentaje de participación del sector hoteles y restaurantes en el producto. Fuente: Elaboración propia con base en datos del DANE. 
la contribución del turismo a la primera variación mencionada.

De acuerdo a la construcción de la metodología los datos deben ser interpretados de la siguiente forma. En el año 2005, la economía antioqueña creció en términos per cápita a una tasa de 2,98\%; de dicho porcentaje 0,04\% puede ser vinculado directamente al crecimiento de las actividades turísticas. De la misma forma deben leerse todas las cifras mencionadas, siempre recordando que los porcentajes de contribución están referidos a la

\begin{tabular}{|l|r|r|r|r|r|r|r|r|}
\hline & 1991 & 1992 & 1993 & 1994 & 1995 & 1996 & 1997 & 1998 \\
\hline Var PBI & 0,66 & 1,14 & 2,79 & 3,46 & 3,16 & $-0,16$ & 1,91 & $-0,96$ \\
\hline Var PBI T & 1,34 & 1,70 & $-0,10$ & 0,90 & 4,08 & $-7,42$ & $-6,99$ & $-0,63$ \\
\hline $\begin{array}{l}\text { Contribución } \\
\text { Turismok }\end{array}$ & 0,04 & 0,05 & 0,00 & 0,02 & 0,11 & $-0,20$ & $-0,17$ & $-0,01$ \\
\hline & & & & & & & & \\
\hline & & 1999 & 2000 & 2001 & 2002 & 2003 & 2004 & 2005 \\
\hline Var PBI & & $-5,48$ & 2,51 & 0,55 & 0,88 & 2,94 & 3,44 & 2,98 \\
\hline Var PBI T & & $-7,56$ & 1,93 & 1,05 & 0,21 & 7,26 & 4,53 & 2,91 \\
\hline Contribución & & & & & & & & \\
Turismo & & $-0,17$ & 0,04 & 0,02 & 0,00 & 0,16 & 0,10 & 0,07 \\
\hline
\end{tabular}

Cuadro 2: Contribución al turismo en Colombia. Fuente: Elaboración propia con base en datos del DANE.

\begin{tabular}{|l|r|r|r|r|r|r|r|r|}
\hline & 1991 & 1992 & 1993 & 1994 & 1995 & 1996 & 1997 & 1998 \\
\hline Variación del PBI & 2,40 & $-4,65$ & 1,55 & 0,75 & 4,33 & $-0,35$ & 2,87 & $-4,65$ \\
\hline Variación del PBI del turismo & $-2,91$ & $-0,58$ & $-0,92$ & 0,17 & $-0,22$ & $-9,75$ & $-2,62$ & $-1,92$ \\
\hline Contribución del turismo & $-0,06$ & $-0,01$ & $-0,02$ & 0,00 & 0,00 & $-0,19$ & $-0,05$ & $-0,03$ \\
\hline & & & & & & & & \\
\hline & & 1999 & 2000 & 2001 & 2002 & 2003 & 2004 & 2005 \\
\hline Variación del PBI & & $-5,48$ & 2,51 & 0,55 & 0,88 & 2,94 & 3,44 & 2,98 \\
\hline Variación del PBI del turismo & & $-11,95$ & 4,23 & 3,72 & 0,49 & 4,26 & 6,22 & 2,40 \\
\hline Contribución del turismo & & $-0,21$ & 0,07 & 0,06 & 0,01 & 0,07 & 0,10 & 0,04 \\
\hline
\end{tabular}

Cuadro 3: Contribución al turismo en el departamento de Antioquía. Fuente: Elaboración propia con base en datos del DANE.

tasa de crecimiento de la economía.

En general, podemos decir que todos los departamentos seleccionados acompañan la tendencia de crecimiento que presenta el país en su conjunto (ver Brida et al. 2009A y 2009B), presentando un aumento del producto hasta 1996 y una reversión de la tendencia a partir de ese momento. Una vez que la economía colombiana retoma su senda de crecimiento hacia el año
2001, los departamentos considerados siguen el mismo camino. En cuanto al producto turístico, tal como ocurre al considerar a Colombia en su conjunto, el PBI turístico acompaña el comportamiento del producto en su conjunto, con una tendencia de crecimiento, la cual se ve interrumpida entre los años 1996 y 2000. Es de destacar el resultado en lo relativo a la contribución del turismo. Hasta 2003, este indicador presenta valores en general bajos en los períodos en los cuales las economías de los diferentes departamentos crecen. Sin embargo, contribuye significativamente a los períodos de recesión de la economía, lo cual da lugar a un resultado interesante que resalta la importancia del sector considerado. Finalmente, en los últimos años, los cuales se caracterizan por un crecimiento de la economía, se observa, tal como ocurre a nivel agregado, un incremento en la contribución del sector turístico al crecimiento económico.

El análisis de las cifras presentadas en los cuadros permite afirmar que en todas las regiones desde el año 2002 la contribución del turismo presenta una tendencia creciente. Hasta el año 2002 la contribución es baja e incluso en algunos casos negativa, aún con tasas de crecimiento de la economía positivas (obsérvese en particular los años 1996 y 1997 para todas las regiones, menos San Andrés y Providencia). Luego del mencionado año, se registra un crecimiento muy importante de la contribución del turismo al crecimiento económico. Por lo tanto puede inferirse del análisis de las cifras presentadas el desarrollo en los últimos años de un sector turístico mucho más dinámico, con mayores aportes al crecimiento y en expansión. Si se comparan los resultados presentados antes con los hallados en (Brida et al, 2008A, 2008B, 2008C) para el caso de Argentina, Brasil, México y Uruguay, se observa que la contribución en media para el caso de las regiones en estudio es mayor que para los tres países de América del Sur, siendo similar al caso mexicano. Por otra parte, la comparación con los países estudiados en Brida et al (2008B) permite concluir que el aporte al crecimiento económico en el caso colombiano es menor a los niveles registrados para España, Italia, Reino Unido y Estados Unidos; siendo similar en algunos años al caso de Francia. 


\begin{tabular}{|l|r|r|r|r|r|r|r|r|}
\hline & 1991 & 1992 & 1993 & 1994 & 1995 & 1996 & 1997 & 1998 \\
\hline Variación del PBI & 1,31 & $-0,26$ & 2,23 & 8,44 & $-0,72$ & $-3,43$ & 0,78 & $-0,22$ \\
\hline Variación del PBI del turismo & 4,73 & 4,88 & 1,67 & 4,50 & 12,27 & $-8,56$ & $-9,18$ & 7,95 \\
\hline Contribución del turismo & 0,09 & 0,09 & 0,03 & 0,09 & 0,23 & $-0,18$ & $-0,18$ & 0,14 \\
\hline & & & & & & & & \\
\hline & & 1999 & 2000 & 2001 & 2002 & 2003 & 2004 & 2005 \\
\hline Variación del PBI & & $-11,56$ & 2,68 & $-0,70$ & 2,48 & 1,75 & 5,42 & 3,18 \\
\hline Variación del PBI del turismo & & $-13,86$ & 6,98 & $-1,78$ & 3,87 & 16,52 & 4,63 & 3,75 \\
\hline Contribución del turismo & & $-0,27$ & 0,13 & $-0,04$ & 0,08 & 0,33 & 0,11 & 0,08 \\
\hline
\end{tabular}

Cuadro 4: Contribución al turismo en Bogotá D.C. Fuente: Elaboración propia con base en datos del DANE.

\begin{tabular}{|l|r|r|r|r|r|r|r|r|}
\hline & 1991 & 1992 & 1993 & 1994 & 1995 & 1996 & 1997 & 1998 \\
\hline Variación del PBI & 12,38 & 3,05 & $-1,59$ & $-1,55$ & 5,79 & $-2,69$ & $-0,44$ & 7,62 \\
\hline Variación del PBI del turismo & 21,60 & $-0,68$ & $-8,10$ & $-5,05$ & 9,42 & $-7,10$ & $-36,06$ & $-2,06$ \\
\hline Contribución del turismo & 1,33 & $-0,05$ & $-0,52$ & $-0,30$ & 0,55 & $-0,43$ & $-2,06$ & $-0,08$ \\
\hline & & & & & & & & \\
\hline & & 1999 & 2000 & 2001 & 2002 & 2003 & 2004 & 2005 \\
\hline Variación del PBI & & $-6,44$ & 5,21 & 0,01 & 6,37 & 6,00 & 8,04 & $-0,51$ \\
\hline Variación del PBI del turismo & & 5,51 & 16,61 & 3,77 & 0,02 & 12,88 & 7,20 & 4,25 \\
\hline Contribución del turismo & & 0,18 & 0,63 & 0,16 & 0,01 & 0,53 & 0,31 & 0,18 \\
\hline
\end{tabular}

Cuadro 5: Contribución al turismo en el departamento de Bolívar. Fuente: Elaboración propia con base en datos del DANE.

\begin{tabular}{|l|r|r|r|r|r|r|r|r|}
\hline & 1991 & 1992 & 1993 & 1994 & 1995 & 1996 & 1997 & 1998 \\
\hline Variación del PBI & $-2,24$ & 7,45 & 8,47 & 2,87 & 5,85 & 2,08 & 2,83 & $-0,36$ \\
\hline Variación del PBI del turismo & 2,54 & 5,73 & 3,78 & 4,67 & 5,22 & $-0,58$ & $-2,33$ & $-0,45$ \\
\hline Contribución del turismo & 0,15 & 0,37 & 0,24 & 0,28 & 0,32 & $-0,04$ & $-0,14$ & $-0,03$ \\
\hline & & & & & & & & \\
\hline & & 1999 & 2000 & 2001 & 2002 & 2003 & 2004 & 2005 \\
\hline Variación del PBI & & $-5,26$ & $-3,58$ & 4,57 & $-0,20$ & 2,82 & 3,07 & 3,29 \\
\hline Variación del PBI del turismo & & $-2,36$ & 0,53 & 9,53 & 2,55 & 2,23 & 4,19 & 3,79 \\
\hline Contribución del turismo & & $-0,13$ & 0,03 & 0,58 & 0,16 & 0,15 & 0,27 & 0,25 \\
\hline
\end{tabular}

Cuadro 6: Contribución al turismo en el departamento de Magadalena. Fuente: Elaboración propia con base en datos del DANE. 


\begin{tabular}{|l|r|r|r|r|r|r|r|r|}
\hline & 1991 & 1992 & 1993 & 1994 & 1995 & 1996 & 1997 & 1998 \\
\hline & & & - & & - & & & \\
Variación del PBI & 9,36 & 9,75 & 14,23 & 4,54 & 11,53 & 11,60 & $-0,83$ & 3,75 \\
\hline Variación del PBI del turismo & $-3,78$ & 7,55 & 6,34 & 6,61 & $-9,45$ & 19,28 & 1,54 & $-3,61$ \\
\hline Contribución del turismo & $-0,73$ & 1,29 & 1,06 & 1,37 & $-2,01$ & 4,19 & 0,36 & $-0,86$ \\
\hline & & & & & & & & \\
\hline & & 1999 & 2000 & 2001 & 2002 & 2003 & 2004 & 2005 \\
\hline & & & & - & & & & \\
Variación del PBI & & $-3,99$ & 9,67 & 17,51 & 11,72 & 6,12 & 1,62 & 3,81 \\
\hline Variación del PBI del turismo & & 15,70 & 0,61 & 6,29 & 5,60 & $-5,40$ & 3,23 & 3,65 \\
\hline Contribución del turismo & & 3,47 & 0,16 & 1,54 & 1,76 & $-1,61$ & 0,86 & 0,98 \\
\hline
\end{tabular}

Cuadro 7: Contribución al turismo en el departamento de San Andrés y

Providencia. Fuente: Elaboración propia con base en datos del DANE.

\section{La hipótesis del turismo como generador de desamollo y crecimiento de largo periodo}

Comenzamos entonces, tal como mencionamos, considerando el grado de integración de las series bajo estudio a partir de la aplicación el test de Dick y Fuller aumentado (ADF). La tabla 1 muestra los resultados de la aplicación del mencionado test, incluyendo tendencia y constante.

En todos los cinco casos bajo estudio encontramos que los test de raíces unitarias en niveles y diferencias indican claramente que las tres series son integradas de orden 1, es decir, aunque las series no son estacionarias, sus primeras diferencias si lo son. A pesar de que en algunos de los casos de la tabla los resultados no indican esta tendencia, al realizar el análisis con tendencia y sin tendencia ni constante-que no incluimos por cuestiones de espacio- se confirma el hecho de que todas las series son integradas de orden 1 .

La tabla 2 muestra el test de cointegracion de Johansen, que identifica para las cinco regiones bajo estudio una relación de cointegracion entre el PIB, el gasto real en turismo (TE) y el tipo de cambio real (TCR). Se observa claramente la existencia de una relación de integración para todas las regiones bajo estudio.

Adicionalmente, en la Tabla 3 mostramos también que la variable TE es débilmente exógena para cada uno de los departamentos. Para Magdalena y para San Andrés y Providencia, el test de verosimilitud indica que no podemos rechazar la hipótesis nula de exogeneidad débil de TE y TCR. Dado que nosotros estamos interesados en las relaciones de largo periodo entre las variables, esto no es relevante La exogeneidad

\begin{tabular}{|c|c|c|c|c|}
\hline Departamento & $\begin{array}{c}\text { Nivel/Primeras } \\
\text { diferencias }\end{array}$ & PBI per cápita & $\begin{array}{l}\text { Gasto en } \\
\text { Turismo }\end{array}$ & TCR \\
\hline Antioquia & Nivel & $\begin{array}{l}-1,84 \\
(0,63) \\
\text { NRH0 }\end{array}$ & $\begin{array}{c}-1,64 \\
0,71 \\
\text { NRHO }\end{array}$ & $\begin{array}{l}-3,21 \\
(0,11) \\
\text { NRHO } \\
\end{array}$ \\
\hline Antioquia & $\begin{array}{l}\text { Primeras } \\
\text { Diferencias }\end{array}$ & $\begin{array}{c}-4,56 \\
(0,01) \\
\text { RHO }\end{array}$ & $\begin{array}{l}-0,93 \\
(0,91) \\
\text { NRHO }\end{array}$ & $\begin{array}{c}-5,94 \\
(0,00) \\
\text { RHO }\end{array}$ \\
\hline Bogotá & Nivel & $\begin{array}{l}-1,52 \\
(0,77) \\
\text { NRHO } \\
\end{array}$ & $\begin{array}{c}-3,64 \\
0,06 \\
\text { NRHO } \\
\end{array}$ & $\begin{array}{l}-3,21 \\
(0,11) \\
\text { NRHO } \\
\end{array}$ \\
\hline Bogotá & $\begin{array}{l}\text { Primeras } \\
\text { Diferencias }\end{array}$ & $\begin{array}{l}-3,21 \\
(0,12) \\
\text { NRHO }\end{array}$ & $\begin{array}{c}-5,45 \\
(0,00) \\
\text { RHO }\end{array}$ & $\begin{array}{c}-5,94 \\
(0,00) \\
\text { RHO }\end{array}$ \\
\hline Bolívar & Nivel & $\begin{array}{l}-1,71 \\
(0,69) \\
\text { NRHO }\end{array}$ & $\begin{array}{c}-1,29 \\
0,84 \\
\text { NRHO }\end{array}$ & $\begin{array}{l}-3,21 \\
(0,11) \\
\text { NRH0 }\end{array}$ \\
\hline Bolívar & $\begin{array}{l}\text { Primeras } \\
\text { Diferencias }\end{array}$ & $\begin{array}{c}-5,02 \\
(0,00) \\
\text { RH0 } \\
\end{array}$ & $\begin{array}{l}-3,16 \\
(0,13) \\
\text { NRHO } \\
\end{array}$ & $\begin{array}{c}-5,94 \\
(0,00) \\
\text { RHO } \\
\end{array}$ \\
\hline Magdalena & Nivel & $\begin{array}{l}-2,95 \\
(0,17) \\
\mathrm{NRHO}\end{array}$ & $\begin{array}{c}-2,99 \\
0,17 \\
\text { NRHO }\end{array}$ & $\begin{array}{l}-3,21 \\
(0,11) \\
\text { NRHO }\end{array}$ \\
\hline Magdalena & $\begin{array}{l}\text { Primeras } \\
\text { Diferencias }\end{array}$ & $\begin{array}{l}-2,80 \\
(0,21) \\
\text { NRHO }\end{array}$ & $\begin{array}{l}-2,47 \\
(0,42) \\
\text { NRHO }\end{array}$ & $\begin{array}{c}-5,94 \\
(0,00) \\
\text { RHO }\end{array}$ \\
\hline San Andrés & Nivel & $\begin{array}{l}-3,57 \\
(0,06) \\
\text { NRHO }\end{array}$ & $\begin{array}{l}-3,93 \\
(0,03) \\
\mathrm{RH} 0\end{array}$ & $\begin{array}{l}-3,21 \\
(0,11) \\
\text { NRHO }\end{array}$ \\
\hline San Andrés & $\begin{array}{l}\text { Primeras } \\
\text { Diferencias }\end{array}$ & $\begin{array}{c}-6,20 \\
(0,00) \\
\text { RHO }\end{array}$ & $\begin{array}{c}-5,34 \\
(0,00) \\
\text { RHO }\end{array}$ & $\begin{array}{c}-5,94 \\
(0,00) \\
\text { RHO }\end{array}$ \\
\hline
\end{tabular}

Tabla 1: Análisis de estacionalidad. 


\begin{tabular}{|c|c|c|c|c|c|}
\hline \multicolumn{3}{|c|}{ Antioquia } & \multicolumn{3}{|c|}{ Bogotá } \\
\hline \multicolumn{3}{|c|}{ Test de la traza } & \multicolumn{3}{|c|}{ Test de la traza } \\
\hline Hypothesis & \begin{tabular}{|l|} 
Trace \\
Statistic
\end{tabular} & \begin{tabular}{|l|} 
Critical \\
Value
\end{tabular} & Hypothesis & \begin{tabular}{|l|} 
Trace \\
Statistic
\end{tabular} & \begin{tabular}{|l|} 
Critical \\
Value
\end{tabular} \\
\hline None $^{*}$ & 62.77216 & 42.91525 & None* $^{*}$ & 57.45971 & 29.79707 \\
\hline At most $1^{*}$ & 29.08995 & 25.87211 & At most 1 & 13.41733 & 15.49471 \\
\hline At most $2^{*}$ & 12.55149 & 12.51798 & At most 2 & 0.302679 & 3.841466 \\
\hline \multicolumn{3}{|c|}{ Test del Valor Propio Máximo } & \multicolumn{3}{|c|}{ Test del Valor Propio Máximo } \\
\hline Hypothesis & \begin{tabular}{|l|} 
Max-Eigen \\
Statistic
\end{tabular} & Critical Value & Hypothesis & \begin{tabular}{|l|} 
Max-Eigen \\
Statistic \\
\end{tabular} & Critical Value \\
\hline None $^{*}$ & 33.68221 & 25.82321 & None* & 44.04238 & 21.13162 \\
\hline At most 1 & 16.53846 & 19.38704 & At most 1 & 13.11465 & 14.26460 \\
\hline At most $2^{*}$ & 12.551 & 12.51798 & At most 2 & 0.302679 & 3.841466 \\
\hline \multicolumn{3}{|c|}{$\begin{array}{l}\text { *denota rechazo de la hipótesis al nivel } \\
0.05\end{array}$} & \multicolumn{3}{|c|}{$\begin{array}{l}\text { *denota rechazo de la hipótesis al nivel } \\
0.05\end{array}$} \\
\hline \multicolumn{3}{|c|}{ Bolívar } & \multicolumn{3}{|c|}{ Magdalena } \\
\hline \multicolumn{3}{|c|}{ Test de la traza } & \multicolumn{3}{|c|}{ Test de la traza } \\
\hline Hypothesis & $\begin{array}{l}\text { Trace } \\
\text { Statistic }\end{array}$ & \begin{tabular}{|l} 
Critical \\
Value
\end{tabular} & Hypothesis & \begin{tabular}{|l|} 
Trace \\
Statistic
\end{tabular} & \begin{tabular}{|l} 
Critical \\
Value
\end{tabular} \\
\hline None $^{*}$ & 93.61487 & 29.79707 & None* ${ }^{*}$ & 55.78793 & 42.91525 \\
\hline At most $1^{*}$ & 16.43610 & 15.49471 & At most 1 & 25.67575 & 25.87211 \\
\hline At most 2 & 3.302245 & 3.841466 & At most 2 & 6.710394 & 12.51798 \\
\hline \multicolumn{3}{|c|}{ Test del Valor Propio Máximo } & \multicolumn{3}{|c|}{ Test del Valor Propio Máximo } \\
\hline Hypothesis & $\begin{array}{l}\text { Max-Eigen } \\
\text { Statistic }\end{array}$ & Critical Value & Hypothesis & \begin{tabular}{|l|} 
Max-Eigen \\
Statistic
\end{tabular} & Critical Value \\
\hline None $^{*}$ & 77.17877 & 21.13162 & None $^{*}$ & 30.11218 & 25.82321 \\
\hline At most 1 & 13.13386 & 14.26460 & At most 1 & 18.96535 & 19.38704 \\
\hline At most 2 & 3.302245 & 3.841466 & At most 2 & 6.710394 & 12.51798 \\
\hline \multicolumn{3}{|c|}{$\begin{array}{l}\text { *denota rechazo de la hipótesis al nivel } \\
0.05\end{array}$} & \multicolumn{3}{|c|}{$\begin{array}{l}\text { *denota rechazo de la hipótesis al nivel } \\
0.05\end{array}$} \\
\hline & \multicolumn{4}{|c|}{ San Andrés y Providencia } & \\
\hline & \multicolumn{4}{|c|}{ Test de la traza } & \\
\hline & Hypothesis & \begin{tabular}{|l|} 
Trace \\
Statistic
\end{tabular} & \multicolumn{2}{|c|}{ Critical value } & \\
\hline & None* & 49.65071 & \multicolumn{2}{|c|}{42.91525} & \\
\hline & At most 1 & 15.76191 & \multicolumn{2}{|c|}{25.87211} & \\
\hline & At most 2 & 4.312569 & \multicolumn{2}{|c|}{12.51798} & \\
\hline & \multicolumn{4}{|c|}{ Test del Valor Propio Máximo } & \\
\hline & Hypothesis & $\begin{array}{l}\text { Max-Eigen } \\
\text { Statistic }\end{array}$ & \multicolumn{2}{|c|}{ Critical value } & \\
\hline & None $^{*}$ & 33.88880 & \multicolumn{2}{|c|}{25.82321} & \\
\hline & At most 1 & 11.44934 & \multicolumn{2}{|c|}{19.38704} & \\
\hline & At most 2 & 4.312569 & \multicolumn{2}{|c|}{12.51798} & \\
\hline & \multicolumn{4}{|c|}{ *denota rechazo de la hipótesis al nivel 0.05} & \\
\hline
\end{tabular}

Tabla 2: Test de Cointegración de Johansen

débil de la variable TE nos permite hacer inferencia con respecto a los efectos del gasto real en turismo en el crecimiento económico de largo periodo.

La relación de cointegración puede ser expresada

$(P I B)_{t}=\beta_{0}+\beta_{1}(T E)_{t}+\beta_{2}(T C R)_{t}+\beta_{3}(\text { trend })_{t}$ como la siguiente ecuación (1):

donde $B 1$ es la elasticidad del PIB respecto al TE y re-

\begin{tabular}{|c|c|c|}
\hline Región & X cuadrado & P-valor \\
\hline Antioquia & 7,431 & 0,024 \\
\hline Bogotá & 3,926 & 0,340 \\
\hline Bolívar & 4,466 & 0,307 \\
\hline Magdalena* & 30,54 & 0,005 \\
\hline $\begin{array}{c}\text { San Andrés and } \\
\text { Providencia * }\end{array}$ & 30.23 & 0,006 \\
\hline *denota rechazo de la hipótesis al nivel 0.01 \\
\hline
\end{tabular}

Tabla 3: Test de Máxima Verosimilitud. Hipótesis nula: Exogeneidad débil para TE y RER.

presenta el porcentaje de crecimiento de largo periodo en el PIB producido por un incremento del 1\% en TE. La Tabla 4 muestra los valores de los paráme-

\begin{tabular}{|l|c|c|c|c|}
\hline Región & & & & \\
\hline Antioquia & 6,9923 & 0,6216 & $-0,0007$ & 0,0094 \\
\hline Bogotá & 7,0864 & 0,6268 & $-0,0004$ & -0.0192 \\
\hline Bolívar & 11,3254 & 0,2062 & 0,0219 & 0,0227 \\
\hline Magdalena & 7,8935 & 0,5657 & $-0,0030$ & - \\
\hline San Andrés & 33,3408 & 1,7990 & 0,0011 & 0,1095 \\
\hline
\end{tabular}

Tabla 4: Parámetros de la ecuación (1)

tros $B 0, B 1,62$ y $B 3$ obtenidos para las diferentes regiones:.

Podemos entonces afirmar que: 1) existe una relación de equilibrio de largo periodo entre el gasto en el turismo y el crecimiento económico para todas las regiones estudiadas; 2) existe una relación causal unidireccional desde el turismo hacia el crecimiento económico para todas las regiones estudiadas. Esto implica que la bien conocida hipótesis del turismo como generador del crecimiento económico (TLGH) no puede ser rechazada para ninguno de los cinco departamentos en estudio. Nótese también que el valor de la elasticidad $B 1$ es considerable para todas las regiones En especial podemos remarcar el caso del departamento de San Andrés y Providencia, con una economía casi de "monocultivo" en el turismo, que presenta una elasticidad de gran magnitud (1.79). Un crecimiento en el gasto en turismo del orden del millón de dólares en San Andrés y Providencia genera un crecimiento del PIB del departamento del orden del 1,8 millones de dólares. Es claro que esto indica que para este departamento en particular, el 
turismo pude ser un factor fundamental de crecimiento económico y desarrollo de la economía de las islas. De todos modos tenemos que notar que no se puede relacionar una alta participación del turismo en el PIB actual con un valor alto de elasticidad. De hecho Bogotá presenta una alta participación del turismo en el PIB y un valor moderado de la elasticidad relativa (B1) mientras que Bolívar presenta una alta participación del turismo en el PIB y un valor bajo de 81 . En realidad cualquier relación entre estos dos parámetros es posible dado que $B 1$ es el producto de dos factores, la razón TE/PIB y la derivada $\partial \mathrm{PIB} / \partial \mathrm{TE}$ y por lo tanto una baja participación TE/PIB puede ser compensada con una variación

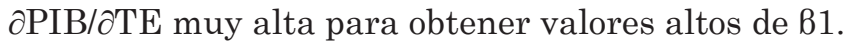

En lo que tiene que ver con el efecto del tipo de cambio real en el PIB de largo periodo, la situación es diferente dependiendo de la región. Para tres departamentos (Antioquia, Bogotá y Magdalena) hay un coeficiente negativo mientras que en Bolívar, San Andrés y Providencia, el PIB y el TCR de largo periodo están positivamente correlacionados.

Si comparamos los resultados obtenidos en este trabajo con otros artículos que investigan las relaciones de largo periodo entre turismo crecimiento y desarrollo económico, vemos que la elasticidad de San Andrés y Providencia es asombrosamente alta, la única que es mayor a 1 entre los estudios de Latino América, aunque se pueden señalar otros casos en economías fuertemente dependientes del turismo. La siguiente tabla resume los resultados para algunos países del continente americano.

En la comparación con los destinos latinoamericanos de la Tabla 5, se puede notar que Bolívar presenta una elasticidad relativamente baja y San Andrés una relativamente alta, mientras que los otros tres departamentos tienen un valor de 81 en la media de los países descriptos. Esto muestra la importancia de hacer un análisis regional y no por países ya que destinos en un mismo país pueden presentar relaciones de largo periodo entre turismo y crecimiento y desarrollo económico disimiles.

\section{Conclusiones}

En el presente artículo hemos estudiado las relaciones entre el sector turismo y el crecimiento económico bajo dos perspectivas, aplicándolas a la economía de los principales destinos turísticos de Colombia. El estudio pretende ser una contribución a la comprensión del impacto del turismo para la economía colombiana. Primero cuantificamos la contribución regional de la industria turística al crecimiento de la economía en las dos últimas décadas y luego estudiamos la importancia del turismo en el crecimiento de largo plazo.

Mostramos que, en el período bajo estudio, los departamentos de Colombia presentan dos períodos bien diferenciados en cuanto a la evolución de peso de las actividades turísticas en el PBI. En la década de los noventa se verifica un descenso de dicha participación del orden de medio punto porcentual del PBI. Luego, a partir del año 2000 hay un incremento sostenido del peso de las actividades turísticas, aunque no llega a los niveles de 1990. Esta relación, que se verifica en la media, se verifica también para cada una de las regiones en estudio. En futuras investigaciones se deberán integrar los datos de las cuentas satélite del turismo para Colom-

\begin{tabular}{|l|c|c|c|l|}
\hline País & Periodo & $\begin{array}{c}\text { Periodicidad de los } \\
\text { datos }\end{array}$ & $\beta_{1}$ & \multicolumn{1}{|c|}{ Articulo } \\
\hline México & $1980-2007$ & cuatrimestral & 0.69 & Brida et al. (2008C) \\
\hline MERCOSUR and Chile & $1990-2000$ & anual & 0.74 & Gardella and Aguayo (2002) \\
\hline Colombia & $1907-2007$ & cuatrimestral & 0.51 & Brida et al. (2009A) \\
\hline $\begin{array}{l}\text { High and medium income } \\
\text { Latin American Countries }\end{array}$ & $1985-1998$ & anual & - & Eugenio-Martin et al. (2004) \\
\hline $\begin{array}{l}\text { Low income Latin American } \\
\text { Countries }\end{array}$ & $1985-1998$ & anual & - & Eugenio-Martin et al. (2004) \\
\hline Chile & $1988-2008$ & anual & 0.81 & Brida et al. (2008B) \\
\hline Uruguay & $1987-2006$ & cuatrimestral & 0.42 & Brida et al. (2008D) \\
\hline
\end{tabular}

Tabla 5: Estudios empíricos acerca de la relación de largo periodo entre turismo y crecimiento económico para algunas economías latinoamericanas. 
bia y sus departamentos (aún no están disponibles) para tener una aproximación al sector turismo más veraz.

Hemos también analizado los efectos del gasto en turismo en el crecimiento económico de los departamentos de Colombia. En todos los cinco casos bajo estudio encontramos que los test de raíces unitarias en niveles y diferencias indican claramente que las tres series son integradas de orden 1, es decir, aunque las series no son estacionarias, sus primeras diferencias si lo son. Seguidamente se aplicó la técnica de Johansen para investigar las relaciones de correlación entre las variables utilizadas: el gasto en turismo, el PBI per cápita y el tipo de cambio real. Estas variables representan indicadores del crecimiento económico de Colombia, del ingreso por turismo internacional y de la competitividad externa. La evidencia empírica obtenida sugiere la existencia de una relación de cointegración entre el PIB per cápita real, el gasto en turismo y el tipo de cambio real para todos los cinco departamentos bajo estudio. El análisis de cointegracion confirma la hipótesis de que las ganancias obtenidas por el turismo afectan positivamente el crecimiento económico de largo período en cada uno de los departamentos. No obstante, los diferentes valores de las elasticidades del PIB respecto al gasto en turismo, sugieren que los efectos de un aumento del gasto en turismo en Colombia no serán iguales para las regiones bajo estudio, siendo San Andrés y Providencia el departamento donde el impacto es mayor (y considerablemente alto) y Bolívar el departamento donde el impacto es menor. Para los otros tres departamentos bajo estudio, mostramos que el valor de la elasticidad está en la media de los valores obtenidos en otros destinos del continente.

El significativo impacto del turismo para la economía de las regiones colombianas estudiadas en este artículo sugiere la necesidad de políticas públicas que apoyen las iniciativas de desarrollo turístico de las tantas potenciales atracciones que tiene el país y que incrementen la demanda turística internacional y doméstica. Colombia tiene la oportunidad de aprender de experiencias precedentes en el resto del mundo, positivas y negativas, para corregir errores de otros destinos (algunos irreversibles) y promover iniciativas que minimicen los impactos del desarrollo del turismo.

\section{Agradecimientos}

Nuestra investigación fue apoyada por la Free University of Bolzano, mediante el proyecto "Tourism, growth, development and sustainability. The case of the South Tyrolean region" y la Institución Universitaria
Colegio Mayor de Antioquia con el proyecto "Políticas, crecimiento económico y turismo. El caso de Antioquia"

\section{Bibliografía}

Balaguer, J. y Cantavella-Jordà, M.

2002 "Tourism as a long-run economic growth factor: the Spanish case". Applied Economics, 34, 877-884.

Banco de la República.

2006 Informe de Coyuntura Económica Regional Departamento de San Andrés Islas [En línea]. Disponible en <http://www.banrep.gov.co/documentos/publicaciones/regional/ICER/san_andres/2006_2.pdf> Consultado el 10 de febrero de 2010.

Brida, J.G., Risso, W.A., Zapata-Aguirre, S., Pereyra, J.S. y Such Devesa, M.J.

2009A "Tourism and economic growth: an empirical analysis for the case of Colombia". TOURISMOS: An International Multidisciplinary Journal of Tourism, 4 (2).

Brida, J.G., Risso, W.A., Zapata-Aguirre, S., Pereyra, J.S. y Such Devesa, M.J.

2009B "Turismo y crecimiento económico: un análisis empírico de Colombia”. Estudios y Perspectivas en Turismo, 18 (1): 21-35.

Brida, J.G., Such, M.J. y Pereira, J.S.

2008 "Evaluating the contribution of tourism in economic growth". Anatolia: an International Journal of Tourism and Hospitality Research, 19(2): 351-356.

Brida, J.G., Zapata-Aguirre, S., Pereyra, J.S. y Such Devesa, M.J.

2008B "La contribución del turismo al crecimiento económico: el caso de España, Francia, Italia, Reino Unido y Estados Unidos". Cuadernos de Turismo, 22, 35-46.

Brida, J.G., Carrera, E., y Risso, W.A.

2008C “Tourism's Impact on Long-run Mexican Economic Growth". Economics Bulletin, 3, (7):1-10.

Brida, J.G., Lanzilotta, B., Risso, W.A.

2008D "Turismo y crecimiento económico: el caso de Uruguay". PASOS: Revista de Turismo y Patrimonio Cultural, 6 (3): 481-492

Capó, J., Riera, A. y Rosselló, J.

2007 "Tourism and long-term growth a Spanish perspective". Annals of Tourism Research, 34(3):709-726. Capó, J., Riera, A. y Rosselló, J.

2006 "Specialising in Tourism and Long-Term Growth". Documents de Treball CRE, 3, 1-21.

Dolando, J.J., Jenkinson, T y Sosvilla, Rivero, S.

1990 "Cointegration and Unit Roots". Journal of Economic Surveys, 4 (3): 249-273. 
Dritsakis, N.

2004 "Tourism as a long-run economic growth factor: an empirical investigation for Greece using causality analysis". Tourism Economics, 10(3):305-316.

Durbarry, R.

2004 "Tourism and economic growth: The case of Mauritius". Tourism Economics, 10, 389-401.

Engle, R. y Granger, C.

1987 "Co-integration and error correction: representation, estimation and testing”. Econometrica, 55, 251276.

Eugenio-Martin, J. L., N. M. Morales, y Scarpa, R.

2004 "Tourism and Economic Growth in Latin American Countries: A Panel Data Approach". Fondazione Eni Enrico Mattei Working Paper Series, Nota di Lavoro 26.

Figuerola, M.

2005 "Turismo y desarrollo regional: Hacia una perspectiva multidisciplinaria". Trabajo presentado en el VII CONGRESO NACIONAL DE INVESTIGACIÓN TURISTICA. Universidad de Guadalajara. México. Disponible en <http://www.sectur.gob.mx/work/sites/sectur/resources/LocalContent/13580/3/Presentacion_ManuelF.pdf>. Consultado el 18 de noviembre de 2009.

Gardella, R. y Aguayo, E.

2002 "Impacto Economico del Turismo en el Mercosur y Chile". Estudios Economicos de Desarrollo Internacional. AEEADE. 2 (1).

Granger, C.

1988 "Some recent developments in a concept of causality”. Journal of Econometrics, 39, 199-211.

Gobernación de Antioquia.

Plan de Desarrollo Antioquia 2008-2011. Disponible en $<$ http://www.antioquia.gov.co/documentos/plandedesarrollo4.pdf $>$ Consultado el 10 de febrero de 2010.

Ivanov, S. y Webster, C.

2007 "Measuring the impacts of tourism on economic growth”. Tourism Economics, 13(3): 379-388.

Johansen, S.

1988 "Statistical Analysis of Cointegration Vectors". Journal of Economic Dynamics and Control, 12, 231254.

Johansen, S. y Juselius, K.

1990 "Maximum likelihood estimation and inference on cointegration with applications to the demand for money". Oxford Bulletin of Economics and Statistics, 52,169-210.

Johansen, S.

1995 Likelihood-based inference in cointegrated vector autorregressive models. Oxford University Press, Oxford.
Kim, H. J., Chen, M. y Jan, S.

2006 "Tourism expansion and economic development: The case of Taiwan”. Tourism Management, 27(5): 925-933.

Meyer, D.

2004 Economía turística en América Latina y el Caribe. Bogotá: Ed. Universidad Externado de Colombia, Facultad de Administración de Empresas Turísticas y Hoteleras.

Noriko, I y Mototsugu, F.

2007 "Impacts of tourism and fiscal expenditure to remote islands: the case of the Amami islands in Japan”. Applied Economics Letter, 14, 661-666.

Organización Mundial del Turismo OMT

2009 Noticias OMT. Año XXIII. Número 2.

Pérez, G.

2005 "Bolívar: industrial, agropecuario y turístico". Centro de Estudios Económicos Regionales (CEER) de Cartagena. Banco de la República. N58, Julio.

Quintero P., Bernal C. y López H.

2005 "La competitividad turística de Cartagena de Indias: Análisis del destino y posicionamiento en el mercado". Serie de Estudios de Competitividad de Cartagena, $\mathrm{N}^{\mathrm{o}} 4$, Cámara de Comercio de Cartagena, Observatorio del Caribe Colombiano y Colciencias, Cartagena de Indias.

Secretaría distrital de Planeación.

2007 Síntesis de coyuntura. Dirección de Políticas Sectoriales. $\mathrm{N}^{\circ} 11$, Bogotá Disponible en http://www.sdp. gov.co/www/resources/No_11_comercio.pdf. Consultado el 24 de noviembre de 2009.

\section{Notas}

1. Política Sectorial de Turismo 2008-2010 “Colombia destino turístico de clase mundial”. Viceministerio de Turismo.

2. En www.legicomex.com/ BancoMedios/Documentos\%20 PDF/exportacionescol.pdf

3. Informe Monitor La Ventaja Competitiva de Medellín. Cámara de Comercio de Medellín.

4. Alcaldía Mayor de Bogotá.

5. En www.mincomercio.gov.co

6. Alcaldía de Santa Marta. Marco conceptual y metodológico para la formulación y elaboración del plan sectorial de turismo de santa marta 2009 - 2019. En: http://santamarta-magdalena. gov.co/

7. En: http://www.sanandres.gov.co/

$\begin{array}{lr}\text { Recibido: } & 16 / 02 / 10 \\ \text { Reenviado: } & 15 / 02 / 11 \\ \text { Aceptado: } & 28 / 01 / 11 \\ \text { Sometido a evaluación por pares anónimos }\end{array}$

